Pacific Journal of Mathematic 


\title{
SCALE-INVARIANT MEASURABILITY IN WIENER SPACE
}

\author{
G. W. Johnson and D. L. Skoug
}

The fact that change of scale is a pathological transformation in Wiener space has long been known. For many problems, this pathology causes no special difficulties. However it is sometimes necessary to consider functions of the form $F(\lambda x)$ where $\lambda$ varies over the positive reals and $x$ varies over Wiener space. In this setting a variety of conceptual subtleties arise. In this paper we give a framework and several results which prove useful in dealing with these difficulties. In the last section of this paper we discuss several papers in the recent literature in the light of this framework.

1. Notation and terminology; introduction. Let $T=[0,1]$ and let $C_{0}(T)$ denote Wiener space, that is, the space of real-valued continuous functions on $T$ which vanish at $t=0$ (The notation $C_{0}(T)$ will never be abbreviated to $C_{0}$. The latter notation will be introduced latter for a certain proper subsets of $C_{0}(T)$.). Let $\mathscr{B}$ denote the Borel measurable subsets of $C_{0}(T)$ and let $m_{1}$ denote Wiener measure. One can complete $\left(C_{0}(T), \mathscr{B}, m_{1}\right)$ in the usual way to obtain $\left(C_{0}(T), \mathscr{S}_{1}, m_{1}\right)$ where $\mathscr{S}_{1}$ is the class of all Wiener measurable sets. Let $\sigma_{n}$ be the partition $0=t_{0}<t_{1}<\cdots<t_{2^{n}}=1$ where $t_{k}=k / 2^{n}$ for $k=0,1, \cdots, 2^{n}$. Given $x$ in $C_{0}(T)$, let $S_{\sigma_{n}}(x)=\sum_{k=1}^{2^{n}}\left[x\left(t_{k}\right)-\right.$ $\left.x\left(t_{k-1}\right)\right]^{2}$. For $\lambda \geqq 0$, let $C_{\lambda} \equiv\left\{x\right.$ in $\left.C_{0}(T): \lim _{n \rightarrow \infty} S_{a_{n}}(x)=\lambda^{2}\right\}$ and let $D \equiv\left\{x\right.$ in $C_{0}(T): \lim _{n \rightarrow \infty} S_{\sigma_{n}}(x)$ fails to exist $\}$. Note that $\lambda C_{\mu}=C_{\lambda \mu}$. Clearly $D$ and the sets $C_{\lambda}, \lambda \geqq 0$, are all Borel sets and $C_{0}(T)$ is the disjoint union of this family of sets.

The key to our discussion is the following result due to Lévy [35] and independently, but later, to Cameron and Martin [8].

Theorem 1. $m_{1}\left(C_{1}\right)=1$.

Lévy actually established a deeper result. He showed that if $\left\{\pi_{n}\right\}$ is any nested sequence of partitions of $[0,1]$ whose norm approaches zero, then $\lim _{n \rightarrow \infty} S_{\pi_{n}}(x)=1$ for $m_{1}$-a.e. $x$ in $C_{0}(T)$. Cameron and Martin [8] and Cameron [2] showed that Theorem 1 has some surprising implications for the scale change and translation transformations in Wiener space.

A subset $A$ of $C_{0}(T)$ is said to be scale-invariant measurable provided $\lambda A$ is in $\mathscr{S}_{1}$ for all $\lambda>0$. A scale-invariant measurable set 
$N$ is said to be scale-invariant null provided $m_{1}(\lambda N)=0$ for all $\lambda>0$. A property which holds except on a scale-invariant null set will be said to hold $s$-almost everywhere (denoted by $s$-a.e.). Cameron and Martin introdduce the concept of scale-invariant null sets in [8]. The classes of scale-invariant measurable and scale-invariant null sets will be denoted by $\mathscr{S}$ and $\mathscr{N}$ respectively. A function $F$ is said to be scale-invariant measurable provided $F$ is defined on a scale-invariant measurable set and $F(\lambda x)$ is Wiener measurable for every $\lambda>0$. Two functions $F$ and $G$ on Wiener space are said to be equivalent $(F \approx G)$ if they agree $s$-a.e.. This notion of equivalence was introduced by Cameron and Storvick in [16]. Closely related notations of equivalence were introduced earlier in [4 and 27].

The present authors (and others) have often been perplexed about the exact nature of the above concepts. As long as one considers a fixed scaling in Wiener space, the scale change pathologies pointed out in [8 and 2] cause no problems. However it is often necessary [3, 4, $9 \sim 19,21,24 \sim 34,38,39]$ to consider functions of the form $F(\lambda x)$ where $x$ is in $C_{0}(T)$ and $\lambda$ varies over the positive reals. (The natural functions to consider are often somewhat more complicated than $F(\lambda x)$; for example, $F\left(\lambda^{-1 / 2} x+\xi\right)$ where $\lambda>0$, $x \in C_{0}(T)$ and $\xi$ is a real number. We will focus our attention on the functions $F(\lambda x)$ since, once, this is understood, it is not difficult to make adjustments to fit the other cases.) Some of the problems that arise with scale changes can be avoided by considering only Borel measurable functions $F$ on the uncompleted Wiener measure space $\left(C_{0}(T), \mathscr{B}, m_{1}\right)$. Unfortunately however, one cannot avoid all the scale change pathologies by restricting attention to Borel measurable $F$. One striking illustration of this is provided by an example and theorem due to Cameron and Storvick [16, pp. 5-7]. They exhibit two Borel measurable functions $F$ and $G$ which agree except on an $m_{1}$-null Borel set and yet their "Fourier-Feynman transforms" are unequal $m_{1}$-a.e.. In contrast, they show that if $F$ and $G$ are equivalent (equal s-a.e.) and if the "Fourier-Feynman transform" of $F$ exists, then the "Fourier-Feynman transform" of $G$ exists and is equivalent to it. In the last section of this paper we will see that related phenomena occur in the setting of the "Feynman integral".

The results in $\S \S 2$ and 3 on scale change and translations turn out to be quite simple when looked at from the right point of view; in spite of this (perhaps because of this) they provide valuable insight into exactly what scale-invariant measurable sets and scaleinvariant null sets are really like and exactly how they compare to Wiener measurable sets and Wiener null sets respectively. These results allow us to expand on and understand better some of the 
old results in [8] and [2]. In addition, as we will discuss in the last section of this paper, they help considerably in understanding several recent papers.

2. Scale-invariant measurable sets, Let $m_{\lambda}$ be the Borel measure given by $m_{\lambda}(B)=m_{1}\left(\lambda^{-1} B\right)$ for $B$ in $\mathscr{B}$. Since $\lambda^{-1} C_{\lambda}=C_{1}$, we see by Theorem 1 that $m_{\lambda}$ is concentrated on the Borel set $C_{\lambda}$; i.e., $m_{\lambda}\left(C_{\lambda}\right)=1$. Let $\mathscr{S}_{\lambda}$ denote the $\sigma$-algebra obtained by completing $\left(C_{0}(T), \mathscr{B}, m_{\lambda}\right)$ and let $\mathscr{N}_{\lambda}$ denote the $m_{\lambda}$-null sets. Note that every subset of $C_{0}(T) \backslash C_{\lambda}$ is in $\mathscr{N}_{\lambda}$.

Proposition 2. (i) $N$ is in $\eta_{\lambda}$ if and only if $\lambda^{-1} N$ is in $\mathscr{N}_{1}$; equivalently, $\mathscr{N}_{\lambda}=\lambda \mathscr{N}_{1}$. (ii) $E$ is in $\mathscr{S}_{\lambda}$ if and only if $\lambda^{-1} E$ is in $\mathscr{S}_{1}$; equivalently, $\mathscr{S}_{\lambda}=\lambda \mathscr{S}_{1}$. (iii) We have $m_{\lambda}(E)=m_{1}\left(\lambda^{-1} E\right)$ for $E$ in $\mathscr{S}_{\lambda}$.

Proof. (i) Let $N$ be in $\mathscr{N}_{\lambda}$. Then $N \subset M$ where $M$ is an $m_{\lambda^{-}}$ null Borel set. Hence $m_{1}\left(\lambda^{-1} M\right)=m_{\lambda}(M)=0$ and so $\lambda^{-1} M$ is an $m_{1^{-}}$ null Borel set. But then $\lambda^{-1} N \subset \lambda^{-1} M$ is in $\mathscr{N}_{1}$. The converse can be shown in essentially the same way.

(ii) Let $E$ be in $\mathscr{S}_{\lambda}$. Then $E=B \cup N$ where $B$ is in $\mathscr{B}$ and $N$ is in $\mathscr{N}_{\lambda}$. Then $\lambda^{-1} N$ is in $\mathscr{N}_{1}$ by (i) and so $\lambda^{-1} E=\lambda^{-1} B \cup \lambda^{-1} N$ is in $\mathscr{S}_{1}$. The rest of (ii) is easily checked.

(iii) Let $E$ be in $\mathscr{S}_{\lambda}$. Then $E=B \cup N$ where $B$ is in $\mathscr{B}$ and $N$ is $m_{\lambda}$-null. Then

$$
m_{\lambda}(E)=m_{\lambda}(B \cup N)=m_{\lambda}(B)=m_{1}\left(\lambda^{-1} B\right)=m_{1}\left(\lambda^{-1} B \cup \lambda^{-1} N\right)=m_{1}\left(\lambda^{-1} E\right) .
$$

Proposition 3. $\mathscr{S}=\bigcap_{\lambda>0} \mathscr{S}_{\lambda} ; \mathscr{N}=\bigcap_{\lambda>0} \cdot \mathscr{N}_{\lambda} ; \mathscr{S}$ is a $\sigma$-algebra of subsets of $C_{0}(T)$.

Proof. Suppose $A$ is in $\mathscr{S}$. Then $\lambda^{-1} A$ is in $\mathscr{S}_{1}$ for every $\lambda>0$. Hence $A$ is in $\lambda \mathscr{S}_{1}=\mathscr{S}_{\lambda}$ for every $\lambda>0$. Hence $\mathscr{S} \subset \bigcap_{\lambda>0} \mathscr{S}_{\lambda}$. Reversing the argument we see that $\bigcap_{\lambda>0} \mathscr{S}_{\lambda} \subset \mathscr{S}$. Hence $\mathscr{S}=$ $\bigcap_{\lambda>0} \mathscr{S}_{\lambda}$. Similarly $\mathscr{N}=\bigcap_{\lambda>0} \mathscr{N}_{\lambda} . \mathscr{S}$ is a $\sigma$-algebra since the intersection of $\sigma$-algebras is a $\sigma$-algebra.

Proposition 4. (i) $E$ is in $\mathscr{S}$ if and only if $E \cap C_{\lambda}$ is in $\mathscr{S}_{\lambda}$ for every $\lambda>0$. for every $\lambda>0$.

(ii) $E$ is in $\mathscr{N}$ if and only if $E \cap C_{\lambda}$ is in $\mathscr{N}_{\lambda}$

Proof. Suppose $E$ is in $\mathscr{S}$. Let $\lambda>0$ be given. $E$ is in $\mathscr{S}_{\lambda}$ and $C_{\lambda}$ is in $\mathscr{B} \subset \mathscr{S}_{\lambda}$ and so $E \cap C_{\lambda}$ is in $\mathscr{S}_{\lambda}$. Conversely, suppose $E \cap C_{\lambda}$ is in $\mathscr{S}_{\lambda}$ for every $\lambda>0$. We wish to show that $E$ is in $\mathscr{S}_{\lambda}$ for every $\lambda>0$. But $C_{0}(T) \backslash C_{\lambda}$ is $m_{\lambda}$-null and so $E=\left(E \cap C_{\lambda}\right) \cup$ $\left(E \cap\left(C_{0}(T) \backslash C_{\lambda}\right)\right)$ is in $\mathscr{S}_{\lambda}$. (ii) is proved in somewhat similar fashion. 
The next theorem is quite simple, but it gives a very useful characterization of $\mathscr{S}$ and $\mathscr{N}$ in that it shows rather well what scale-invariant measurable sets and scale-invariant null sets are really like and how they compare to Wiener measurable sets and Wiener null sets respectively.

Theorem 5. (i) $E$ is in $\mathscr{S}$ if and only if $E$ has the form

$$
E=\left(\bigcup_{\lambda>0} E_{\lambda}\right) \cup L
$$

where each $E_{\lambda}$ is an $m_{\lambda}$-measurable subset of $C_{\lambda}$ and $L$ is an arbitrary subset of $C_{0} \cup D$. Further, for $E$ written in this manner, $m_{\lambda}(E)=m_{\lambda}\left(E_{\lambda}\right)$ for all $\lambda>0$. (ii) $N$ is in $\mathscr{N}$ if and only if $N$ has the form

$$
N=\left(\bigcup_{\lambda>0} N_{\lambda}\right) \cup L
$$

where each $N_{\lambda}$ is an $m_{\lambda}-n u l l$ subset of $C_{\lambda}$ and $L$ is an arbitrary subset of $C_{0} \cup D$.

Proof. (i ) Suppose $E$ is in $\mathscr{S}$. Let $E_{\lambda}=E \cap C_{\lambda}$ and let $L=$ $E \cap\left(C_{0} \cup D\right)$. The decomposition follows from Proposition 4 and the fact that $C_{0}(T)$ is the disjoint union of $D$ and the sets $C_{\lambda}, \lambda \geqq 0$. Conversely, suppose that $E$ has the form (1). To show that $E$ is in $\mathscr{S}$, it suffices by Proposition 3 to show that $E$ is in $\mathscr{S}_{\lambda_{0}}$ for every $\lambda_{0}>0$. Now $E \cap C_{\lambda_{0}}$ is in $\mathscr{S}_{\lambda_{0}}$ by assumption and $\left(\bigcup_{\substack{\lambda>0 \\ \lambda \neq \lambda_{0}}} E_{\lambda}\right) \subset L$ is $m_{\lambda_{0}}$-null. Hence $E$ is in $\mathscr{S}_{\lambda_{0}}$ as desired. The formula $m_{\lambda}(E)=m_{\lambda}\left(E_{\lambda}\right)$ follows from the fact that $m_{\lambda}$ is concentrated on $C_{\lambda}$.

(ii) If $N$ is in $\mathscr{N}$, then $N$ is in $\mathscr{S}$ and so by (i) can be written in the form $N=\left(\bigcup_{\lambda>0} N_{\lambda}\right) \cup L$ where each $N_{\lambda}$ is in $\mathscr{S}_{\lambda}$ and $L \subset$ $\left(D \cup C_{0}\right)$. We only need to show that $m_{\lambda}\left(N_{\lambda}\right)=0$ for every $\lambda>0$. But $N$ in $\mathscr{N}$ implies, by Propostiion 3 , that $N$ is in $\mathscr{N}_{\lambda}$. Hence $m_{\lambda}(N)=m_{\lambda}\left(N_{\lambda}\right)=0$ as desired. The converse of (ii) is easily checked.

REMARK. The preceding theorem shows rather strikingly that there are many more Wiener measurable sets than scale-invariant measurable sets: $A$ set $E$ is Wiener measurable if and only if it has the form $E_{1} \cup L$ where $E_{1}$ is an $m_{1}$-measurable subset of $C_{1}$ and $L$ is an arbitrary subset of $\left(\cup_{0<\lambda \neq 1} C_{\lambda}\right) \cup D \cup C_{0}$. Similarly a set is Wiener null if and only if it has the form $N_{1} \cup L$ where $N_{1}$ is a $m_{1}$-null subset of $C_{1}$ and $L$ is an arbitrary subset of $\left(\mathrm{U}_{0<\lambda \neq 1} C_{\lambda}\right) \cup$ $D \cup C_{0}$.

Let $0<t_{1}<\cdots<t_{n} \leqq 1$ and let $G$ be a Lebesgue measurable 
subset of $n$-dimensional Euclidean space $\boldsymbol{R}^{n}$. It is well known that sets of the form

$$
E \equiv\left\{x \text { in } C_{0}(T):\left(x\left(t_{1}\right), \cdots, x\left(t_{n}\right)\right) \in G\right\}
$$

are Wiener measurable. (In fact $E$ is Wiener measurable if and only if $G$ is Lebesgue measurable [39].) And that they are not Borel measurable if $G$ is not Borel measurable. It is easy to see that such sets $E$ are scale-invariant measurable since, for any $\lambda>0$, $\lambda E=\left\{x \in C_{0}(T):\left(x\left(t_{1}\right), \cdots, x\left(t_{n}\right)\right) \in \lambda^{-1} G\right\}$ is Wiener measurable.

\section{Proposition 6. For every $\lambda_{0}>0, \mathscr{B} \varsubsetneqq \mathscr{S} \varsubsetneqq \mathscr{S}_{\lambda_{0}}$.}

Proof. The containments are clear from the fact that $\mathscr{B} \subset \mathscr{S}_{\lambda}$ for every $\lambda>0$ and the equality $\mathscr{S}=\bigcap_{\lambda>0} \mathscr{S}_{\lambda}$. Let $G \subset \boldsymbol{R}^{n}$ be Lebesgue measurable but not Borel measurable. Then $E$ as in (3) is in $\mathscr{S}$ but not in $\mathscr{B}$. To see that $\mathscr{S} \neq \mathscr{S}_{\lambda_{0}}$, take $\lambda_{1} \neq \lambda_{0}$ and let $E_{\lambda_{1}}$ be a subset of $C_{\lambda_{1}}$ which is not $m_{\lambda_{1}}$-measurable. Then $E_{\lambda_{1}}$ is in $\mathscr{S}_{\lambda_{0}}$ but not in $\mathscr{S}_{\lambda_{1}}$ and so not in $\mathscr{S}$.

The following striking result of Cameron and Martin [8] becomes rather transparent using Theorem 5 .

COROLlaRY 7. Let $f$ be any function (however nasty) from $(0, \infty)$ to $[0,1]$. Then there exists $E$ in $\mathscr{S}$ such that $m_{1}(\lambda E)=f(\lambda)$ for all $\lambda>0$.

Proof. For each $\lambda>0$, pick $E_{\lambda} \subset C_{\lambda}$ such that $E_{\lambda}$ is in $\mathscr{S}_{\lambda}$ and $m_{\lambda}\left(E_{\lambda}\right)=f\left(\lambda^{-1}\right)$. Then $E \equiv \bigcup_{\lambda>0} E_{\lambda}$ is the desired set since by Proposition 2 and Theorem 5 we have

$$
m_{1}(\lambda E)=m_{\lambda^{-1}}(E)=m_{\lambda^{-1}}\left(E_{\lambda^{-1}}\right)=f(\lambda) .
$$

Our sets $C_{\lambda}, \lambda \geqq 0$, and $D$ depend on the particular sequence of partitions that we choose. If $\pi=\left\{\pi_{1}, \pi_{2}, \cdots\right\}$ denotes another nested sequence of partitions whose norms go to zero, we may let $C_{\lambda}^{\pi} \equiv$ $\left\{x \in C_{0}(T): \lim _{n \rightarrow \infty} S_{\pi_{n}}(x)=\lambda^{2}\right\}$ and $D^{\pi} \equiv\left\{x \in C(T): \lim _{n \rightarrow \infty} S_{\pi_{n}}(x)\right.$ fails to exist\}. Essentially because of Lévy's more general form of Theorem 1 , all of the results obtained up to this point, with changes in notation where appropriate, go through. Note however that $\mathscr{S}_{\lambda}$, $\mathscr{N}_{\lambda}, m_{\lambda}, \mathscr{S}$ and $\mathscr{N}$ are all independent of the sequence of partitions. A set $E$ in $\mathscr{S}$ now has two decompositions according to the two versions of Theorem 5:

$$
E=\left(\bigcup_{\lambda>0} E_{\lambda}\right) \cup L=\left(\bigcup_{\lambda>0} E_{\lambda}^{\pi}\right) \cup L^{\pi}
$$


where $E_{\lambda}^{\pi}=E \cap C_{\lambda}^{\pi}$ and $L^{\pi}=E \cap\left(C_{0}^{\pi} \cup D^{\pi}\right)$. How do these two decompositions relate to one another? The next proposition shows that they agree up to a scale-invariant null set.

Proposition 8. Let $E$ be in $\mathscr{S}$. Then the two decompositions of $E$ given by (4) and corresponding respectively to our original sequence of partitions and to $\pi$ have the property that the set

$$
\left(\bigcup_{\lambda>0} E_{\lambda} \triangle E_{\lambda}^{\pi}\right) \cup\left(L \triangle L^{\pi}\right)
$$

is scale-invariant null.

Proof. First note that for all $\lambda>0$

$$
\begin{aligned}
m_{\lambda}\left(E_{\lambda} \backslash E_{\lambda}^{\pi}\right) & =m_{\lambda}\left[\left(E \cap C_{\lambda}\right) \backslash\left(E \cap C_{\bar{\lambda}}^{\pi}\right)\right] \\
& =m_{\lambda}\left[E \cap\left(C_{\lambda} \backslash C_{\lambda}^{\pi}\right)\right] \\
& \leqq m_{\lambda}\left[C_{\lambda} \backslash C_{\lambda}^{\pi}\right] \\
& \leqq m_{\lambda}\left[C_{0}(T) \backslash C_{\lambda}^{\pi}\right]=0 .
\end{aligned}
$$

Thus by Theorem 5 , the set $\bigcup_{\lambda>0}\left(E_{\lambda} \backslash E_{\lambda}^{\pi}\right) \cup\left(L \backslash L^{\pi}\right)$ is scale-invariant null. In similar fashion one can show that the set $\bigcup_{\lambda>0}\left(E_{\lambda}^{\pi} \backslash E_{\lambda}\right) \cup$ $\left(L^{\pi} \backslash L\right)$ is scale-invariant null which concludes the proof since

$$
\begin{aligned}
\bigcup_{\lambda>0}\left(E_{\lambda} \Delta E_{\bar{\lambda}}^{\tilde{\lambda}}\right) \cup\left(L \Delta L^{\pi}\right) \\
\quad=\left\{\bigcup_{\lambda>0}\left(E_{\lambda} \backslash E_{\bar{\lambda}}^{\bar{\lambda}}\right) \cup\left(L \backslash L^{\pi}\right)\right\} \cup\left\{\bigcup_{\lambda>0}\left(E_{\bar{\lambda}}^{\bar{\lambda}} \backslash E_{\lambda}\right) \cup\left(L^{\bar{\pi}} \backslash L\right)\right\} .
\end{aligned}
$$

3. Translations. In [2] Cameron used the pathology of scale change transformations in Wiener space to show that almost no translations preserve Wiener measurability. Specifically, he obtained a set $E$ in $\mathscr{S}_{1}$ such that $T_{y} E \equiv E+y$ is not in $\mathscr{S}_{1}$ for $m_{1}$-a.e. $y$ in $C_{0}(T)$. We obtain several facts below which fill in this picture. For example, we will see that if $E$ is in $\mathscr{S}_{\sqrt{2}}$ then the set $E+y$ is in $\mathscr{S}_{1}$ for $m_{1}$-a.e. $y$. More generally, we will see that if $E$ is in $\mathscr{S}_{\sqrt{p^{2}+q^{2}}}$, then $E+y$ is in $\mathscr{S}_{p}$ for $m_{q}$-a.e. $y$. We need the following result.

THEOREM 9. Let $p$ and $q$ be positive numbers. The following assertions are equivalent:

(a) $f\left(\sqrt{p^{2}+q^{2}} z\right)$ is an $m_{1}$-measurable function of $z$.

(b) $f(z)$ is an $m_{\sqrt{p^{2}+q^{2}}}$-measurable function of $z$.

(c) $f(x+y)$ is an $m_{p} \times m_{q}$-measurable function of $x$ and $y$.

(d) $f(p x+q y)$ is an $m_{1} \times m_{1}$-measurable function of $x$ and $y$. If any one (and hence all) of (a) $\sim(\mathrm{d})$ holds, then 


$$
\begin{aligned}
\int_{C_{0}(T)} f\left(\sqrt{p^{2}+q^{2}} z\right) d m_{1}(z) & \stackrel{*}{=} \int_{C_{0}(T)} f(z) d m_{\sqrt{p^{2}+q^{2}}}(z) \\
& \stackrel{*}{=} \int_{C_{0}(T) \times C_{0}(T)} f(x+y) d\left(m_{p} \times m_{q}\right)(x, y) \\
& \stackrel{*}{=} \int_{C_{0}(T) \times C_{0}(T)} f(p x+q y) d\left(m_{1} \times m_{1}\right)(x, y)
\end{aligned}
$$

where by $\stackrel{*}{=}$ we mean that if either side exists, both sides exist and they are equal.

Comments on the proof of Theorem 9. A simple use of the change of variables formula [20, p. 163] shows the equivalence of (a) and (b) and the equivalence of (c) and (d) and the corresponding integration formulas. The integration formulas for Borel measurable $f$ were known to Lévy. The fact that (a) implies (d) and the corresponding integration formula is a corollary of a more general result due to Bearman [1]; this corollary was specifically pointed out in [15; Lemma 2, p. 239]. The fact that (d) implies (a) requires some work and may not have appeared in the literature; it was proved in [23]. We omit the proof since we do not need this particular implication below.

REMARK. There is a natural extension of Theorem 9 involving $n$ positive numbers instead of 2 .

The next result follows immediately from Theorem 9 , the fact that for Wiener integrals $\int_{C_{0}(T)} F(-x) d m_{1}(x)=\int_{C_{0}(T)} F(x) d m_{1}(x)$, and the Fubini theorem, if we take $f$ to be the characteristic function of $E$ where $E$ is in $\mathscr{S}_{\sqrt{p^{2}+q^{2}}}$.

THEOREM 10. Let $p$ and $q$ be positive numbers. Let $E$ belong to $\mathscr{S}_{\sqrt{p^{2}+q^{2}}}$. Then $E+y$ and $E-y$ are in $\mathscr{S}_{p}$ for $m_{q}$-a.e. $y$ and $m_{p}(E+y)$ and $m_{p}(E-y)$ are $m_{p}$-measurable functions of $y$. Similarly $E+x$ and $E-x$ are in $\mathscr{S}_{q}$ for $m_{p}$-a.e. $x$ and $m_{q}(E+x)$ and $m_{q}(E-x)$ are $m_{p}$-measurable functions of $x$. Furthermore

$$
\begin{aligned}
\int_{C_{0}(T)} m_{p}(E+y) d m_{q}(y) & =\int_{C_{0}(T)} m_{p}(E-y) d m_{q}(y) \\
& =\left(m_{p} \times m_{q}\right)(\{(x, y): x+y \text { is in } E\}) \\
& =m_{\sqrt{p^{2}+q^{2}}}(E) \\
& =\int_{C_{0}(T)} m_{q}(E-x) d m_{p}(x) \\
& =\int_{C_{0}(T)} m_{q}(E+x) d m_{p}(x) .
\end{aligned}
$$


CoROLlaRY 11. Let $E$ be in $\mathscr{S}_{\sqrt{2}} ;$ then $E+y$ is in $\mathscr{S}_{1}$ for $m_{1-}$ a.e. $y$.

When $p=q=1$, the next corollary is Cameron's earlier result.

COROLlaRY 12. Let $p$ and $q$ be positive numbers. The translation map $T_{y}$ from $\left(C_{0}(T), \mathscr{S}_{q}, m_{q}\right)$ to $\left(C_{0}(T), \mathscr{S}_{p}, m_{p}\right)$ is $m_{q}$-almost never measurability preserving.

Proof. Applying Theorem 10 with $E \equiv C_{\sqrt{p^{2}+q^{2}}}$, we see that $m_{p}\left(y+C_{\sqrt{p^{2}+q^{2}}}\right)=1$ for $m_{q}$-a.e. $y$. Let $M$ be a $m_{p}$-nonmeasurable subset of $C_{p}$. For each $y$ such that $m_{p}\left(y+C_{\sqrt{p^{2}+q^{2}}}\right)=1$, let $M^{*}=$ $M \cap\left(y+C_{\sqrt{p^{2}+q^{2}}}\right)$. Then $M^{*}$ is $m_{p}$-nonmeasurable. Let $A=M^{*}-y$. Since $m_{q}\left(C_{\sqrt{ } \sqrt{p^{2}+q^{2}}}\right)=0$ and $A \subset C_{\sqrt{ }} \overline{p^{2}+q^{2}}$, we see that $A$ is in $\mathscr{N}_{q}$ and hence in $\mathscr{S}_{q}$. But $y+A=M^{*}$ is not in $\mathscr{S}_{p}$.

CoRollary 13. $\left(m_{p} \times m_{q}\right)\left(\left\{(x, y)\right.\right.$ in $C_{0}(T) \times C_{0}(T): x+y$ is in $\left.\left.C_{\sqrt{p^{2}+q^{2}}}\right\}\right)=1$; in particular, $x+y$ is in $C_{\sqrt{2}}$ for $m_{1} \times m_{1}$-a.e. $(x, y)$. In contrast, $\left(m_{p} \times m_{q}\right)\left(\left\{(x, y)\right.\right.$ in $C_{0}(T) \times C_{0}(T): x+y$ is in $C_{\lambda}, \lambda \neq$ $\left.\left.\sqrt{p^{2}+q^{2}}\right\}\right)=0$.

Next we give some positive results concerning the translation of scale-invariant measurable sets and scale-invariant null sets.

Corollary 14. Let $E$ be in $\mathscr{S}$. Then for each $p>0, E+y$ is in $\mathscr{S}_{p}$ with the exception of at most a scale-invariant null sets of $y$ 's.

Proof. Let $E$ be in $\mathscr{S}$ and let $p>0$ be given. It suffices to show that for each $\lambda>0, E+y$ is in $\mathscr{S}_{p}$ for $m_{\lambda}$-a.e. $y$. But $E$ in $\mathscr{S}$ implies that $E$ is in $\mathscr{S}_{\sqrt{p^{2}+\lambda^{2}}}$ and so the result follows from Theorem 10.

Corollary 15. Let $N$ be in $\mathscr{N}$. Then for each $p>0, N+y$ is in $\mathscr{N}_{p}$ with the exception of at most a scale-invariant null set of $y$ 's.

Proof. Let $N$ be in $\mathscr{N}$ and let $p>0$ be given. It suffices to show that for each $\lambda>0, m_{p}(N+y)=0$ for $m_{\lambda}$-a.e. $y$. But $N$ in $\mathscr{N}$ implies that

$$
\int_{C_{0}(T)} m_{p}(N+y) d m_{\lambda}(y)=m_{\sqrt{p^{2}+\lambda^{2}}}(N)=0 .
$$

Open questions. (i) Suppose $E$ is in $\mathscr{S}$. Is it the case that 
$E+y$ is in $\mathscr{S}$ for $s$-a.e. $y$ ? (ii) Suppose $N$ is in $\mathscr{N}$. Is it the case that $N+y$ is in $\mathscr{N}$ for $s$-a.e. $y$ ?

Remark on (ii). Let $N$ be in $\mathscr{N}$. For each $\lambda>0$ let $A_{\lambda}=$ $\left\{y \in C_{0}(T): m_{\lambda}(N+y)>0\right\}$. We know that $A_{\lambda}$ is in $\mathscr{N}$ for each $\lambda>0$. The answer to question (ii) would be yes if one could show that $A \equiv \mathrm{U}_{\lambda>0} A_{\lambda}$ was in $\mathscr{N}$.

Although it will not concern us in this paper, we should mention that there is a small ( $m_{1}$-measure 0$)$ but useful set of translators for which the translation map on $\left(C_{0}(T), \mathscr{S}_{1}, m_{1}\right)$ is measurable. The relevant result is known as the Cameron-Martin translation theorem. For information on this topic, see the papers of Cameron and Martin $[6,7]$, Maruyama [36] and Segal [37].

4. Scale-invariant measurable functions, In this section we give some simple but useful results about measurable functions. Let $\mathscr{B}(F)\left(\mathscr{S}(F), \mathscr{S}_{\lambda}(F) ; \lambda>0\right)$ denote the class of all real-valued functions which are defined on a Borel (scale-invariant measurable, $m_{\lambda^{-}}$ measurable respectively) subset of $C_{0}(T)$ and which are measurable with respect to the $\sigma$-algebra $\mathscr{B}\left(\mathscr{S}, \mathscr{S}_{\lambda}\right.$ respectively). We will only discuss real-valued functions for convenience. However, all the results hold for complex-valued functions as well. The next three propositions follows from Proposition 3, Theorem 9, and Proposition 6 respectively.

Proposition 16. $\mathscr{S}(F)=\bigcap_{\lambda>0} \cdot \mathscr{S}_{\lambda}(F)$.

Proposition 17. Let $F$ be a function defined on a subset $\triangle$ of $C_{0}(T)$. Given $\lambda>0$, let $F_{\lambda}$ be defined on $\lambda^{-1} \Delta$ by $F_{\lambda}(x)=F(\lambda x)$. (i) $F_{\lambda}$ is in $\mathscr{S}_{1}(F)$ if and only if $F$ is in $\mathscr{S}_{\lambda}(F)$. (ii) $F$ is in $\mathscr{S}(F)$ if and only if $F_{\lambda}$ is in $\mathscr{S}_{1}(F)$ for every $\lambda>0$; that is, $\mathscr{S}(F)$ is exactly the class of scale-invariant measurable functions defined in $\S 1$.

Proposition 18. For every $\lambda_{0}>0$, $\mathscr{B}(F) \varsubsetneqq \mathscr{S}(F) \varsubsetneqq \mathscr{S}_{\lambda_{0}}(F)$.

THEOREM 19. Let $F$ be a function with domain $\Delta . \quad F$ is s-a.e. defined and in $\mathscr{S}(F)$ if and only if, for each $\lambda>0,\left.F^{\lambda} \equiv F\right|_{\Delta \cap c_{\lambda}}$ is $m_{\lambda}$-a.e. defined and in $\mathscr{S}_{\lambda}(F)$.

Proof. Suppose $F$ is $s$-a.e. defined and in $\mathscr{S}(F)$. Then by Theorem $5, F$ is defined except on a scale-invariant null set $N=$ 
$\left(\bigcup_{\lambda>0} N_{\lambda}\right) \cup L$ where $N_{\lambda} \subset C_{\lambda}$ with $m_{\lambda}\left(N_{\lambda}\right)=0$ and where $L \subset D \cup C_{0}$. Hence $F^{\lambda}$ is defined on $C_{\lambda}$ except on $N_{\lambda}$ and so $F^{\lambda}$ is $m_{\lambda}$-a.e. defined. Since $F$ is in $\mathscr{S}(F)$, given a Borel subset $B$ of the reals, $F^{-1}(B)$ is in $\mathscr{S}=\bigcap_{\lambda>0} \cdot \mathscr{S}_{\lambda}$. Hence $\left(F^{\lambda}\right)^{-1}(B)=F^{-1}(B) \cap C_{\lambda}$ is in $\mathscr{S}_{\lambda}$. Hence $F^{\lambda}$ is in $\mathscr{S}_{\lambda}(F)$.

Conversely, suppose that for each $\lambda>0, F^{\lambda}$ is defined except on an $m_{\lambda}$-null set $N_{\lambda} \subset C_{\lambda}$ and $F^{\lambda}$ is in $\mathscr{S}_{\lambda}(F)$. Then $F$ must be defined except on some subset of the scale-invariant null set $\left(\cup_{i>0} N_{\lambda}\right) \cup\left(D \cup C_{0}\right)$. Hence $C_{0}(T) \backslash \Delta$ is scale-invariant null and $F$ is $s$-a.e. defined. Let $B$ be a Borel subset of the real line. To show that $F^{-1}(B)$ is in $\mathscr{S}$ it suffices by Proposition 4 to show that $F^{-1}(B) \cap C_{\lambda}$ is in $\mathscr{S}_{\lambda}$ for every $\lambda>0$. But this is so since $F^{-1}(B) \cap C_{\lambda}=\left(F^{\lambda}\right)^{-1}(B)$ which is in $\mathscr{S}_{\lambda}$ as desired.

If $F$ is a bounded, Borel measurable function on the reals and if $\left\{\lambda_{n}\right\}, \lambda$ are positive numbers such that $\lambda_{n} \rightarrow \lambda$, then one easily sees that $\int_{a}^{b} F\left(\lambda_{n} z\right) d z \rightarrow \int_{a}^{b} F(\lambda z) d z$. It is tempting to try to make the same argument for functions on Wiener space, but the following example shows that this cannot be done and further illustrates the care that must be taken in dealing with scale changes.

EXAMPLE 20. Let $\lambda>0$ be given and suppose that $\lambda_{n} \uparrow \lambda$. Let $F(z)=1-\chi_{C_{\lambda}}(z) . \quad F$ is bounded and Borel measurable. Now $z$ is in $C_{1}$ for $m_{1}$-a.e. $z$ and so $\lambda_{n} z$ is in $\lambda_{n} C_{1}=C_{\lambda_{n}} \subset C_{0}(T) \backslash C_{2}$ for $m_{1}$-a.e. $z$. Hence $\int_{C_{0}(T)} F\left(\lambda_{n} z\right) d m_{1}(z)=1$ for every $n$. On the other hand, $\int_{C_{0}(T)} F(\lambda z) d m_{1}(z)=0$ and so

$$
\int_{C_{0}(T)} F\left(\lambda_{n} z\right) d m_{1}(z) \nrightarrow \int_{C_{0}(T)} F(\lambda z) d m_{1}(z) .
$$

A positive result along these lines can be obtained by assuming that $F$ is continuous $s$-a.e..

The next two propositions are easy consequences of Theorem 9.

Proposition 21. Let $F$ be a scale-invariant measurable function. Then for each $p>0, F(p x+y)$ is an $m_{1}$-measurable function of $x$ for s-a.e. $y$.

Proof. Given $p$ we must show that

$\left\{y: F(p x+y)\right.$ fails to be $m_{1}$-measurable as a function of $\left.x\right\}$ is in $\mathscr{N}$. Hence by Proposition 4, given $q>0$ we must show that $\left\{y\right.$ in $C_{q}: F(p x+y)$ fails to be $m_{1}$-measurable as a function of $\left.x\right\}$ is in $\mathscr{N}_{q}$. Since $C_{q}=q C_{1}$ it suffices to show that 
$\left\{y\right.$ in $C_{1}: F(p x+q y)$ fails to be $m_{1}$-measurable as a function of $\left.x\right\}$

is in $\mathscr{N}_{1}$. But $F\left(\sqrt{p^{2}+q^{2}} z\right)$ is an $m_{1}$-measurable function of $z$ by assumption. Hence by Theorem $9, F(p x+q y)$ is an $m_{1} \times m_{1}$-measurable function of $(x, y)$. Hence for $m_{1}$-a.e. $y$ or, equivalently, for $m_{1}$ a.e. $y$ in $C_{1}, F(p x+q y)$ is an $m_{1}$-measurable function of $x$. Hence

$\left\{y\right.$ in $C_{1}: F(p x+q y)$ fails to be $m_{1}$-measurable as a function of $\left.x\right\}$ is in $\mathscr{N}_{1}$ as desired.

Proposition 22. Let $F$ and $G$ be scale-invariant measurable functions that are equal s-a.e.; that is $F \approx G$. Then for every $p, q>0, F(p x+q y)=G(p x+q y)$ for $m_{1} \times m_{1}$-a.e. $(x, y)$.

One can see that several useful functions are $s$-a.e. defined and scale-invariant measurable by starting with the simple proposition that follows.

Proposition 23. Let $0<t_{1}<t_{2}<\cdots<t_{n} \leqq 1$ and let $f$ be $a$ Lebesgue measurable function on $\boldsymbol{R}^{n}$. Let $F(x)=f\left(x\left(t_{1}\right), \cdots, x\left(t_{n}\right)\right)$. Then $F$ is s-a.e. defied and scale-invariant measurable.

Proof. It is well-known that if $f$ is a.e. defined and Lebesgue measurable, then $F$ is $m_{1}$-a.e. defined and Wiener measurable. The result follows since, for every $\lambda>0$, multiplication by $\lambda$ followed by $f$ is a. e. defined and Lebesgue measurable just as $f$ is.

5. Some related papers. In this section we indicate some ways in which the work in this paper provides insight into several recent papers. Except for the first topic below, we will assume that the reader is familiar with the basic definitions. We begin with a discussion of the analytic Wiener and analytic Feynman "integrals" since in this case the necessary definitions can be easily and quickly given. These integrals were introduced by Cameron in [3] and have played a key role in certain later work [4, 16, 34].

Let $\boldsymbol{C}^{+}$denote the set of complex numbers with positive real part. Let $F$ be a function such that the Wiener integral $J(\lambda)=$ $\int_{C_{0}(T)} F\left(\lambda^{-1 / 2} x\right) d m_{1}(x)$ exists for a.e. $\lambda>0$. If there exists a function $J^{*}(\lambda)$ analytic in $C^{+}$such that $J^{*}(\lambda)=J(\lambda)$ for a.e. $\lambda>0$, then we define this "essential analytic extension" of $J$ to be the analytic Wiener integral of $F$ over $C_{0}(T)$ with parameter $\lambda$. For $\lambda$ in $C^{+}$, we write

$$
\int_{C_{0}(T)}^{a_{n w}} F(x) d m_{1}(x)=J^{*}(\lambda)
$$


Let $q$ be a real number $(q \neq 0)$ and let $F$ be a function whose analytic Wiener integral exists in $\boldsymbol{C}^{+}$. Then if the following limit exists, we call it the analytic Feyman integral of $F$ over $C_{0}(T)$ with parameter $q$, and we write

$$
\int_{C_{0}(T)}^{a n f_{q}} F(x) d m_{1}(x)=\lim _{\substack{\lambda \rightarrow-i q \\ \lambda \text { in } C^{+}}} \int_{C_{0}(T)}^{a n w \lambda} F(x) d m_{1}(x) .
$$

Let $G \equiv 0$. Clearly the analytic Wiener and Feynman integrals of $G$ are 0 for all values of the parameters.

THEOREM 24. There exists $F$ such that $F=G(G \equiv 0) m_{1}$-a.e. but

$$
\int_{C_{0}(T)}^{a n w_{\lambda}} F(x) d m_{1}(x) \neq 0 \text { for all } \lambda \text { in } C^{+},
$$

and

$$
\int_{C_{0}(T)}^{a n_{q}} F(x) d m_{1}(x) \neq 0 \text { for all real } q \neq 0 .
$$

In fact, given any subset $A$ of $(0, \infty)$ of Lebesgue measure 0 (for example, the rationals), there exists $F$ such that for every $\lambda$ in $A$, $F\left(\lambda^{-1 / 2} x\right)=0 m_{1}$-a.e. but (6) and (7) hold.

Proof. For the first assertion we take $F(x) \equiv \chi_{C_{0}(T) \backslash C_{1}}(x)$. Then $F(x)=G(x)$ m. $m_{1}$-a.e. since $F=G$ on $C_{1}$. However for $\lambda \neq 1$, $F\left(\lambda^{-1 / 2} x\right)=1 m_{1}$-a.e. and so

$$
\int_{C_{0}(T)}^{a_{n} w_{\lambda}} F(x) d m_{1}(x)=1=\int_{C_{0}(T)}^{a n f_{q}} F(x) d m_{1}(x)
$$

for all $\lambda$ in $C^{+}$and all real $q \neq 0$.

To prove the second assertion, let $F(x) \equiv \chi_{C_{0}(T) \cup\langle\in \Lambda} C_{\lambda^{-1 / 2}}(x)$. For $\lambda_{0}$ in $\Lambda, F\left(\lambda_{0}^{-1 / 2} x\right)=0$ for $m_{1}$-a.e. $x$ siuce $\lambda_{0}^{-1 / 2} x$ is in $\lambda_{0}^{-1 / 2} C_{1}=C_{\lambda_{0}^{-1 / 2}}$ for $m_{1}$-a.e. $x$. For $\lambda_{0}$ not in $\Lambda, F\left(\lambda_{0}^{-1 / 2} x\right)=1$ for $m_{1}$-a.e. $x$. Since $A$ has measure 0 , we again have (8) for all $\lambda$ and $q$.

The following positive result follows easily from the definitions and our earlier considerations.

THEOREM 25. Suppose that $\Lambda$ is a subset of $(0, \infty)$ of Lebesgue measure 0 and that for $\lambda$ not in $\Lambda, H\left(\lambda^{-1 / 2} x\right)=F\left(\lambda^{-1 / 2} x\right)$ for $m_{1}$-a.e. $x$. Then if $\int_{C_{0}(T)}^{a n w_{\lambda}} F(x) d m_{1}(x)$ exists throughout $C^{+}, \int_{C_{0}(T)}^{a n w_{\lambda}} H(x) d m_{1}(x)$ exists throughout $C^{+}$and equality holds. Also if $\int_{C_{0}(T)}^{a n_{f_{q}}} F(x) d m_{1}(x)$ exists, then $\int_{C_{0}(T)}^{a n f_{q}} H(x) d m_{1}(x)$ exists and equality holds. 
CoRollary 26. If $H \approx F$, the conclusions of Theorem 25 holds.

When they exists, $\int_{C_{0}(T)}^{a n w_{\lambda}} F(x) d m_{1}(x)$ is an analytic function in $C^{+}$ and the Feynman integrals $\int_{C_{0}(T)}^{a n f_{q}} F(x) d m_{1}(x)$ are the boundary values of this analytic function. It is natural to ask if arbitrary analytic functions and their boundary values can arise as analytic Wiener and Feynman integrals. They can as our next result shows.

THEOREM 27. Given an arbitrary analytic function $g$ on $\boldsymbol{C}^{+}$, there exists a scale-invariant measurable function $F$ such that for all $\lambda$ in $\boldsymbol{C}^{+}$

$$
\int_{C_{0}(T)}^{a n w_{\lambda}} F(x) d m_{1}(x)=g(\lambda)
$$

Proof. Let $F(x)=g(\lambda)$ for $x$ in $C_{\lambda^{-1 / 2}}, \lambda>0$ and let $F(x)=0$ for $x$ in $C_{0} \cup D . \quad F$ is scale-invariant measurable by Theorem 19 . The result now follows since for $\lambda>0$,

$$
\int_{C_{0}(T)} F\left(\lambda^{-1 / 2} x\right) d m_{1}(x)=\int_{C_{1}} F\left(\lambda^{-1 / 2} x\right) d m_{1}(x)=\int_{C_{1}} g(\lambda) d m_{1}(x)=g(\lambda) .
$$

The next result shows that one can get arbitrary analytic functions $g$ (and so arbitrary boundary values) even with very "nasty" $F$ 's.

THEOREM 28. Given any subset $\Lambda$ of $(0, \infty)$ of Lebesgue measure 0 and any analytic function $g$ on $C^{+}$there exists a function $F$ such that $F\left(\lambda^{-1 / 2} x\right)$ fails to be $m_{1}$-measurable for all $\lambda$ in $\Lambda$ but $\int_{C_{0}(T)}^{a n w_{\lambda}} F(x) d m_{1}(x)=g(\lambda)$ for all $\lambda$ in $C^{+}$.

Proof. We define $F$ as in Theorem 27 except that for $F$ in $C_{\lambda-1 / 2}, \lambda$ in $\Lambda$, we let $F(x)=\chi_{A_{\lambda}}(x)$ where $A_{\lambda}$ is an $m_{\lambda^{-1 / 2}}$-nonmeasurable subset of $C_{\lambda-1 / 2}$. Since $\Lambda$ has measure $0, \int_{C_{0}(T)}^{a n w_{\lambda}} F(x) d m_{1}(x)=g(\lambda)$ just as before, but of course $F\left(\lambda^{-1 / 2} x\right)$ is $m_{1}$-nonmeasurable for all $\lambda$ in $\Lambda$.

We have just seen that arbitrary analytic functions $g$ can arise as analytic Wiener integrals. The next theorem is along opposite lines.

THEOREM 29. Given an arbitrary function $f$ on $(0, \infty)$ (however nasty), there exists a scale-invariant measurable function $F$ such that for all $\lambda>0$ 


$$
\int_{C_{0}(T)} F\left(\lambda^{-1 / 2} x\right) d m_{1}(x)=f(\lambda)
$$

Proof. Let $F(x)=f(\lambda)$ for $\lambda$ in $C_{\lambda-1 / 2}, \lambda>0$ and let $F(x)=0$ for $x$ in $C_{0} \cup D$ and argue as in Theorem 27.

We next turn to a discussion of the papers [16] and [34] on the Fourier-Feynman transform. We commented in $\S 1$ on Cameron and Storvick's example [16] of Borel measurable functions $F$ and $G$ such that $F=G m_{1}$-a.e. but their Fourier-Feynman transforms $T_{q} F$ and $T_{q} G$ are unequal $m_{1}$-a.e. The functions used by Cameron and Storvick were $F \equiv 1$ and $G=\chi_{C_{1}}$. We remark that the same functions provide a similar kind of example in the setting of the $L_{p}$ Fourier-Feynman transform [34].

In [16] and [34] all the functions considered are required to be Borel measurable. However a study of the proofs shows that for the class of functions dealt with in detail by those papers, namely functions $F$ as in Proposition 23 and others "built" out of such functions, Lebesgue measurability rather than Borel measurability is the "right" assumption on the functions $f$. On the other hand, it is clear from the Cameron-Storvick example and other considerations that Wiener measurability is not the appropriate setting for these papers. It turns out that the concept of scale-invariant measurability is precisely the correct one for the theory developed in [16] and [34]. In I IV below we describe the main results of [16] in the scale-invariant measurability setting.

I. If $F$ is scale-invariant measurable and if $T_{q} F$ (the $L_{2}$ analytic Fourier-Feynman transform of $F$ ) exists, then $T_{q} F$ is scale-invariant measurable. If, in addition, $G$ is scale-invariant measurable and $G \approx F$, then $T_{q} G$ exists and $T_{q} G \approx T_{q} F$.

II. Let $F$ be as in Proposition 23 with $f$ Lebesgue measurable and in $L_{2}\left(\boldsymbol{R}^{n}\right)$. Then $T_{q} F$ exists and is scale-invariant measurable.

III. Let $\Delta_{n} \equiv\left\{\left(t_{1}, \cdots, t_{n}\right): 0<t_{1}<\cdots<t_{n} \leqq 1\right\}$. Let $f$ be a Lebesgue measurable function on $\Delta_{n} \times \boldsymbol{R}^{n}$ such that $f\left(t_{1}, \cdots, t_{n} ; \cdot, \cdots, \cdot\right)$ is in $L_{2}\left(\boldsymbol{R}^{n}\right)$ for a.e. $\left(t_{1}, \cdots, t_{n}\right)$ in $\Delta_{n}$ and $\sup _{\Delta_{n}}\left\|f\left(t_{1}, \cdots, t_{n} ; \cdot, \cdots, \cdot\right)\right\|_{2}<\infty$. Let

$$
F(x)=\int \cdots \int f\left(t_{1}, \cdots, t_{n} ; x\left(t_{1}\right), \cdots, x\left(t_{n}\right)\right) d t_{1} \cdots d t_{n} .
$$

Then $F$ is defined $s$-a.e. and is scale-invariant measurable. Furthermore $T_{q} F$ exists and is scale-invariant measurable. 
IV. One can proceed to build a larger space by taking certain sums of functions as in III. One can show that such functions $F$ are scale-invariant measurable and that $T_{q} F$ exists and is scaleinvariant measurable. We remark that this final class of functions includes functions of the form. $F(x)=\exp \left(\int_{0}^{1} \theta(t, x(t)) d t\right)$, a class of functions on Wiener space which is of considerable interest.

We will not give proofs of $\mathrm{I} \sim \mathrm{IV}$ above. We note however that Proposition 21 plays a key role in the proof of I and Propositions 21 and 23 play major roles in the proof of II. II is then used to prove III which, in turn, is used to prove IV. Finally we remark that improvements similar to I $\sim$ IV can be made throughout [34].

Next we give some discussion and results associated with the operator-valued Feynman integral or Cameron-Storvick function space integral as studied in $[9 \sim 13,18,19,21,24 \sim 33]$. These results clarify the basic definitions of the theory in a variety of ways and show the necessity of the equivalences introduced in $[27,29,30,33]$. The results below hold in the $L\left(L_{p}, L_{p^{\prime}}\right)$ theory [33] in general and for all allowable dimensions. However, for simplicity, we give the results just in the $L\left(L_{2}, L_{2}\right)$ case with dimension 1 . See [9] or [33] for the basic definitions.

One considers complex-valued functions $F$ on $C(T)$. Any $y$ in $C(T)$ has a unique decomposition $y=x+\xi$ where $x$ is in $C_{0}(T)$ and $\xi$ is in $\boldsymbol{R}$. Hence it is natural to regard two functions $F$ and $G$ on $C(T)$ as equivalent if $F(x+\xi)=G(x+\xi)$ for a.e. $(x, \xi)$ in $C_{0}(T) \times \boldsymbol{R}$. However we will see that this is not the right notion of equivalence for the operator-valued function space integrals $I_{\lambda}(F)$. Let $G$ be identically 0 on $C(T)$. Given any $p>0, p \neq 1$, we will show in Corollary 31 below that there exists a function $F$ on $C(T)$ such that $F(x+\xi)=G(x+\xi)$ for a.e. $(x, \xi)$ but $I_{p}(G)$ is the 0 operator whereas $I_{p}(F)$ is the operator $K_{p}$ in $L\left(L_{2}, L_{2}\right)$ defined by

$$
\left(K_{p} \psi\right)(\xi)=(p / 2 \pi)^{1 / 2} \int_{-\infty}^{\infty} \psi(x) \exp \left(-p(x-\xi)^{2} / 2\right) d x
$$

(See [33, Lemma 1.1] and [24, p. 776] for some discussion concerning this operator; in those papers it was denoted by $C_{\lambda}$.)

THEOREM 30. Let $Z$ be an arbitrary subset of $(0, \infty)$ and let $W=(0, \infty) \backslash Z$. Then there exists a function $F_{Z}$ on $C(T)$ such that $I_{\lambda}\left(F_{Z}\right)$ exists as a bounded linear operator on $L_{2}(R)$ for every $\lambda>0$ and

$$
I_{\lambda}\left(F_{Z}\right)=\left\{\begin{array}{lr}
0 & \text { operator, } \lambda \text { in } Z \\
K_{\lambda} & , \lambda \text { in } W
\end{array}\right.
$$


Also for every $\lambda$ in $Z$ and for every $\xi$ in $R, F_{Z}\left(\lambda^{-1 / 2} x+\xi\right)=0$ for $m_{1}$-a.e. $x$ in $C_{0}(T)$ while for every $\lambda$ in $W$ and for every $\xi$ in $R$, $F_{Z}\left(\lambda^{-1 / 2} x+\xi\right)=1$ for $m_{1}$-a.e. $x$.

Proof. Let $F_{Z}(y)=\chi_{U_{W} c_{\lambda-1 / 2}}(y-y(0))$. Then for every $\lambda_{0}>0$,

$$
\begin{aligned}
F_{Z}\left(\lambda_{0}^{-1 / 2} x+\xi\right) & =\chi_{W} c_{\lambda-1 / 2}\left(\lambda_{0}^{-1 / 2} x+\xi-\lambda_{0}^{-1 / 2} x(0)-\xi\right)=\chi_{W} c_{\lambda-1 / 2}\left(\lambda_{0}^{-1 / 2} x\right) \\
& =\chi_{W} \lambda_{J}^{1 / 2} c_{\lambda-1 / 2}(x)=\chi_{W} c_{\lambda_{0}^{1 / 2}}^{1 / 2-1 / 2}(x) .
\end{aligned}
$$

Now for $\lambda_{0}$ in $W, C_{1}$ is one of the sets $\left\{C_{\lambda_{0}^{1 / 2} \lambda_{-1 / 2}}: \lambda\right.$ in $\left.W\right\}$ and so, for every $\xi$ in $\boldsymbol{R}, F_{Z}\left(\lambda_{0}^{-1 / 2} x+\xi\right)=1$ for $m_{1}$-a.e. $x$. Thus for $\psi \in L_{2}(\boldsymbol{R})$

$$
\begin{aligned}
\left(I_{\lambda_{0}}\left(F_{Z}\right) \psi\right)(\xi) & =\int_{C_{0}(T)} F_{Z}\left(\lambda_{0}^{-1 / 2} x+\xi\right) \psi\left(\lambda_{0}^{-1 / 2} x(1)+\xi\right) d m_{1}(x) \\
& =\int_{C_{0}(T)} \psi\left(\lambda_{0}^{-1 / 2} x(1)+\xi\right) d m_{1}(x) \\
& =\left(K_{\lambda_{0}} \psi\right)(\xi)
\end{aligned}
$$

as was to be shown. Now let $\lambda_{0}$ be in $Z$. Then for every $\xi$ in $\boldsymbol{R}$, $F_{Z}\left(\lambda_{j}^{-1 / 2} x+\xi\right)=0$ for $m_{1}$-a.e. $x$. Thus

$$
\left(I_{\lambda_{0}}\left(F_{Z}\right) \psi\right)(\xi)=\int_{C_{0}(T)}(0) \psi\left(\lambda_{0}^{-1 / 2} x(1)+\xi\right) d m_{1}(x)=0 .
$$

Note that $\left.F_{Z}\right|_{c_{0}(T)}$ is $\mathscr{S}$-measurable. In fact, if either $Z$ or $W$ is countable, it is clear that $\left.F_{Z}\right|_{C_{0}(T)}$ is Borel measurable.

CoROllaRy 31. Let $Z$ be any subset of $(0, \infty)$ such that 1 is in $Z$. Then $F_{Z}(x+\xi)=G(x+\xi)$ for a.e. $(x, \xi)$. However for every $\lambda$ in $W, I_{\lambda}\left(F_{Z}\right)=K_{\lambda}$ whereas $I_{\lambda}(G)$ is the 0 operator. Moreover, if $W \neq \phi, I_{\lambda}^{a n}\left(F_{z}\right)$ and $J_{q}^{a n}\left(F_{Z}\right)$ fail to exist whereas $I_{\lambda}^{a n}(G)$ and $J_{q}^{a n}(G)$ are all the 0 operators.

Proof. We just need to be comment on the last assertion. It is well-known that $K_{\lambda}$ is analytic in $\lambda$ throughout $C^{+}$[24] and is never the 0 operator. For $I_{\lambda}^{a n}\left(F_{z}\right)$ to exist it would have to be an operator-valued analytic function on $C^{+}$such that, (i) $I_{\lambda}^{a n}\left(F_{Z}\right)=I_{\lambda}\left(F_{Z}\right)$ is the zero operator for $\lambda$ in $Z$ and, (ii) $I_{\lambda}^{a n}\left(F_{Z}\right)=I_{\lambda}\left(F_{Z}\right)=K_{\lambda}$ for $\lambda$ in $W$. But this cannot happen by the Identity theorem [22] since at least one of $Z, W$ has a limit point in $(0, \infty)$. Since $I_{\lambda}^{a n}\left(F_{Z}\right)$ fails to exist, the operators $J_{q}^{a n}\left(F_{Z}\right)$ cannot possibly exist.

In the next corollary, we explicitly point out that it is possible to have $F_{Z}$ equal to $G$ in a very strong sense and yet fail to have $I_{\lambda}\left(F_{Z}\right)=I_{\lambda}(G)$ for some $\lambda>0$. 
Corollary 32. Let $p>0, p \neq 1$ and let $W=\{p\}$. Then for every $\lambda>0, \lambda \neq p$, and for every $\xi$ in $R, F_{z}\left(\lambda^{-1 / 2} x+\xi\right)=G\left(\lambda^{-1 / 2} x+\xi\right)$ for $m_{1}$-a.e. $x$. However $I_{p}\left(F_{z}\right)=K_{p}$ whereas $I_{p}(G)=0$. Further the operators $I_{\lambda}^{a n}\left(\boldsymbol{F}_{Z}\right)$ and $J_{q}^{a n}\left(\boldsymbol{F}_{Z}\right)$ fail to exist.

REMARK. If one defined $I_{\lambda}^{a n}(F)$ in a slightly different but reasonable way, $I_{\lambda}^{a n}\left(F_{z}\right)$ would exist and be the 0 operator for the $F_{Z}$ from Corollary 32. This could be accomplished by defining $I_{\lambda}^{a n}(F)$ as an operator-valued analytic function on $C^{+}$which agrees with $I_{\lambda}(F)$ for a.e. $\lambda$ in $(0, \infty)$. Even with this altered definition, one could still get $I_{\lambda}^{a n}\left(F_{Z}\right)$ failing to exist simply by taking $Z$ so that both $Z$ and $W$ have nonzero measure.

The following positive results are easily established.

THEOREM 33. Let $F$ and $H$ be functions on $C(T)$.

(a) Fix $\lambda>0$ and suppose that $I_{\lambda}(F)$ exists and that $H\left(\lambda^{-1 / 2} x+\xi\right)=F\left(\lambda^{-1 / 2} x+\xi\right)$ for a.e. $(x, \xi)$. Then $I_{\lambda}(H)$ exists and equals $I_{\lambda}(F)$.

(b) Suppose $I_{\lambda}^{a n}(F)$ exists for $\lambda$ in $C^{+}$and that, for every $\lambda>0$, $H\left(\lambda^{-1 / 2} x+\xi\right)=F\left(\lambda^{-1 / 2} x+\xi\right)$ for a.e. $(x, \xi)$. Then $I_{\lambda}^{a n}(H)$ exists throughout $C^{+}$and equals $I_{\lambda}^{a n}(F)$.

(c) Suppose $J_{q}^{a n}(F)$ exists for $q \neq 0$ in $R$ and that for every $\lambda>0, H\left(\lambda^{-1 / 2} x+\xi\right)=F\left(\lambda^{-1 / 2} x+\xi\right)$ for a.e. $(x, \xi)$. Then $J_{q}^{a n}(H)$ exists and equals $J_{q}^{a n}(F)$.

$I_{\lambda}^{a n}(F)$, when it exists, is an operator-valued analytic function of $\lambda$ for $\lambda$ in $\boldsymbol{C}^{+}$. Is there anything special about the analytic functions that arise in this way? For example, can one put any limits on the growth of $\left\|I_{\lambda}^{a n}(F)\right\|$ as $\lambda \rightarrow-i q$ ? The following result answers some such questions in the negative.

THEOREM 34. Let $g(\lambda)$ be an arbitrary scalar-valued analytic function on $C^{+}$. Then there exists $F$ such that $I_{\lambda}^{a n}(F)$ exists and $I_{\lambda}^{a n}(F)=g(\lambda) K_{\lambda}$ for $\lambda$ in $C^{+}$. For this $F,\left\|I_{\lambda}^{a n}(F)\right\|=|g(\lambda)|$ for all $\lambda$ in $\boldsymbol{C}^{+}$.

Proof. $g(\lambda) K_{\lambda}$ is an operator-valued analytic function of $\lambda$ for $\lambda$ in $C^{+}$and so it suffices to find $F$ such that $I_{\lambda}(F)=g(\lambda) K_{\lambda}$ for $\lambda>0$. Let

$$
F(y)= \begin{cases}g(\lambda) & \text { when } y-y(0) \text { is in } C_{\lambda^{-1 / 2}}, \lambda>0 \\ 0 & \text { when } y-y(0) \text { is in } C_{0} \cup D .\end{cases}
$$

Now for $\lambda>0, \lambda^{-1 / 2} x+\xi-\lambda^{-1 / 2} x(0)-\xi=\lambda^{-1 / 2} x$ which is in $C_{\lambda^{-12}}$ 
for all $\xi$ and $m_{1}$-a.e. $x$. Hence for each $\lambda>0,\left(\lambda^{-1 / 2} x+\xi\right)=g(\lambda)$ for every $\xi$ and $m_{1}$-a.e. $x$. Hence

$$
\begin{aligned}
\left(I_{\lambda}(F) \eta^{\prime}\right)(\xi) & =\int_{C_{0}(T)} F\left(\lambda^{-1 / 2} x+\xi\right) \psi\left(\lambda^{-1 / 2} x(1)+\xi\right) d m_{1}(x) \\
& =g(\lambda) \int_{C_{0}(T)} \psi\left(\lambda^{-1 / 2} x(1)+\xi\right) d m_{1}(x) \\
& =g(\lambda)\left(K_{\lambda} \psi\right)(\xi) .
\end{aligned}
$$

The result now follows since $\left\|K_{\lambda}\right\|=1[24$, p. 776$]$.

The next result shows that $I_{\lambda}(F), \lambda>0$, can depend on $\lambda$ in a rather arbitrarily pathological way.

THEOREM 35. Let $f(\lambda)$ be any function (however nasty) on $(0, \infty)$. Then there exists $F$ such that $I_{\lambda} F=f(\lambda) K_{\lambda}$ for $\lambda>0$.

Proof. Simply let

$$
F(y)= \begin{cases}f(\lambda) & \text { when } y-y(0) \text { is in } C_{\lambda}^{-1 / 2}, \lambda>0, \\ 0 & \text { when } y-y(0) \text { is in } C_{0} \cup D,\end{cases}
$$

and argue as in the preceding proof.

We will conclude this section with some relatively brief comments on the papers $[14,15,17]$. Although we are less familiar with these papers, it appears very likely that some of the ideas and results of this paper (as well as the extensions of [16] found in [34] in the case $p=2$ ) will allow one to better understand and extend these papers as well. As a test of this, we tried to see if we could improve on Theorem 1 of [15] with reference to the assumptions on $\gamma$. In [15], it is assumed that $\psi$ is Borel measurable and that for every $\lambda>0$ and every $\eta$ in $C_{0}(T), \psi(\lambda y+\eta)$ is Wiener integrable as a function of $y$. We get a related result under the simpler and more general assumption that $\psi$ is scale-invariant integrable; i.e., $\psi$ is $s$-a.e. defined and $\mathscr{S}$-measurable and $\int_{C_{0}(T)}|\psi(\lambda x)| d m_{1}(x)<\infty$ for every $\lambda>0$.

THEOREM 36. Let $F$ be bounded continuous in the uniform topology on $C_{2}^{*}[R]$ and let $\lambda>0$. Then the operator $I_{\lambda}(F)$. (now defined in terms of a Yeh-Wiener integral) carries the space of scale-invariant integrable functions into itself.

We will not carry out the proof but we mention that the key step involves a use of Proposition 21 and an extension of Theorem 1 of [14[ to a situation summarized by the formula 


$$
\begin{aligned}
& \int_{C_{2}[R] \times C_{1}[\alpha, \beta]} f(x(b, \cdot), y(\cdot)) d\left(m \times m_{1}\right)(x, y) \\
& \quad=\int_{C_{1}[\alpha, \beta] \times C_{1}[\alpha, \beta]} f\left(((b-a) / 2)^{1 / 2} z(\cdot), y(\cdot)\right) d\left(m_{1} \times m_{1}\right)(z, y)
\end{aligned}
$$

where $m$ denotes Yeh-Wiener measure on $C_{2}[R]$.

In addition to the likelihood of being able to extend [17], it appears that one should be able to give examples and results for the operator-valued Yeh-Feynman integral which parallel the examples and results given earlier in this section for the operator-valued Cameron-Storvick (or Feynman) integral.

\section{REFERENCES}

1. J. E. Bearman, Rotations in the product of two Wiener spaces, Proc. Amer. Math. Soc., 3 (1952), 129-137.

2. R. H. Cameron, The translation pathology of Wiener space, Duke Math. J., 21 (1954), 623-628.

3. - A family of integrals serving to connect the Wiener and Feynman integrals, J. of Math. and Physics, 39 (1960), 126-140.

4. — The Ilstow and Feynman integrals, J. Anal. Math., 10 (1962/1963), 287361 .

5. R. H. Cameron and R. E. Graves, Additive functionals on a space of continuous functions, Trans. Amer. Math. Soc., 70 (1951), 160-176.

6. R. H. Cameron and W. T. Martin, Transformations of Wiener integrals under translations, Ann. Math., 45 (1944), 386-396.

7. - Transformations of Wiener integrals under a general class of linear transformations, Trans. Amer. Math. Soc., 58 (1945), 184-219.

8. - The behavior of measure and measurability under change of scale in Wiener space, Bull. Amer. Math. Soc., 53 (1947), 130-137.

9. R. H. Cameron and D. A. Storvick, An operator valued function space integral and a related integral equation, J. Math. and Mech., 18 (1968), 517-552.

10. - An integral equation related to the Schroedinger equation with application to integration in function space, Problems in Analysis, Princeton U. Press, Princeton, (1970), 175-193.

11. - An operator valued function space integral applied to integrals of functions of class $L_{2}$, J. Math. Anal. Appl., 42 (1973), 330-372.

12. - An operator valued function space integral applied to integrals of functions of class $L_{1}$, Proc. London Math. Soc., 27 (1973), 345-360.

13. - An operator valued function space integral applied to multiple integrals of functions of class $L_{1}$, Nagoya Math. J., 51 (1973), 91-122.

14. - Two related integrals over spaces of continuous functions, Pacific J. Math., 55 (1974), 19-37.

15. - An operator valued Yeh-Wiener integral and a Wiener integral equation, Indiana, Univ. Math. J., 25 (1976), 235-258.

16. — An $L_{2}$ analytic Fourier-Feynman transform, Michigan Math. J., 23 (1976), 1-30.

17. — An operator-valued Yeh-Feynman integral and a Feynman integral equation, to appear.

18. R. J. Collins, An operator valued function space integral applied to analytic and non-analytic functions of integrals, Thesis, U. of Minnesota, (1971). 
19. Robert A. Ewan, The Cameron-Storvick operator valued function space integral for a class of finite dimensional functionals, Thesis, U. of Nebraska, (1972).

20. P. Halmos, Measure Theory, Van Nostrand, Princeton, New Jersey, 1950.

21. Bernard O. Haugsby, An operator valued integral in a function space of continuous vector valued functions, Thesis, U. of Minnesota, (1972).

22. E. Hille and R. S. Phillips, Functional Analysis and Semi-groups, Amer. Math. Soc. Colloq. Publ., 31 (1957).

23. G. W. Johnson, unpublished class notes.

24. G. W. Johnson and D. L. Skoug, Operator-valued Feynman integrals of certain finite-dimensional functionals, Proc. Amer. Math. Soc., 24 (1970), 774-780.

25. - Operator-valued Feynman integrals of finite-dimensional functionals, Pacific J. Math., 34 (1970), 774-780.

26. - An operator valued function space integral: a sequel to Cameron and Storvick's paper, Proc. Amer. Math. Soc., 27 (1971), 514-518.

27. — A Banach algebra of Feynman integrable functionals with application to an integral equation formally equivalent to Schroedinger's equation, J. of Functional Analysis, 12 (1973), 129-152.

28. — Feynman integrals of non-factorable finite-dimensional functionals, Pacific J. Math., 45 (1973), 257-267.

29. - Cameron and Storvick's function space integral for certain Banach spaces of functionals, J. of London Math. Soc., 9 (1974), 103-117.

30. - Cameron and Storvick's function space integral for a Banach space of functionals generated by finite-dimensional functionals, Annali Di Mathematica Pura Ed Applicata, 104 (1975), 67-83.

31. — A function space integral for a Banach space of functionals on Wiener space, Proc. Amer. Math. Soc., 43 (1974), 141-148.

32. - The Cameron-Storvick function space integral: The $L_{1}$ theory, J. of Math. Anal. and Appl., 50 (1975), 647-667.

33. - The Cameron-Storvick function space integral: an $L\left(L_{p}, L_{p^{\prime}}\right)$ theory, Nagoya J. Math., 60 (1976), 93-137.

34. — An $L_{p}$ analytic Fourier-Feynman transform, to appear in Michigan Math. J.

35. P. Lévy, Le mouvement Brownien plan, Amer. J. Math., 62 (1940), 487-550.

36. G. Maruyama, Notes on Wiener integrals, Kodai Mathematical Seminar Reports, 3 (1950), 41-44.

37. I. E. Segal, Distributions in Hilbert space and canonical systems of operators, Trans. Amer. Math. Soc., 88 (1958), 12-41.

38. D. L. Skoug, Generalized Ilstow and Feynman integrals, Pacific J. Math., 26 (1968), 171-192.

39. Converses to measurability theorems for Yeh-Wiener space, Proc. Amer. Math. Soc., 57 (1976), 304-310.

40. - The change of scale and translation pathology in Yen-Wiener space, Riv. Mat. Univ. Parma, 3 (1977), 78-87.

Received October 19, 1978 and in revised form December 20, 1978.

UNIVERSITY OF NEBRASKA

LINCOLN, NE 68588 


\section{PACIFIC JOURNAL OF MATHEMATICS}

EDITORS

DONALD BABBITT (Managing Editor)

University of California

Los Angeles, California 90024

Hugo RossI

University of Utah

Salt Lake City, UT 84112

C. C. MOORE and ANDREW OGG

University of California

Berkeley, CA 94720

\section{J. DUGUNDJI}

Department of Mathematics University of Southern California Los Angeles, California 90007

R. Finn and J. Milgram Stanford University

Stanford, California 94305

\section{ASSOCIATE EDITORS}
E. F. BECKENBACH
B. H. Neumann
F. WOLF
K. YoSHIDA

\section{SUPPORTING INSTITUTIONS}

UNIVERSITY OF BRITISH COLUMBIA

CALIFORNIA INSTITUTE OF TECHNOLOGY

UNIVERSITY OF CALIFORNIA

MONTANA STATE UNIVERSITY

UNIVERSITY OF NEVADA, RENO

NEW MEXICO STATE UNIVERSITY

OREGON STATE UNIVERSITY

UNIVERSITY OF OREGON
UNIVERSITY OF SOUTHERN CALIFORNIA

STANFORD UNIVERSITY

UNIVERSITY OF HAWAII

UNIVERSITY OF TOKYO

UNIVERSITY OF UTAH

WASHINGTON STATE UNIVERSITY

UNIVERSITY OF WASHINGTON 


\section{Pacific Journal of Mathematics}

\section{Vol. 83, No. 1 \\ March, 1979}

Richard Neal Ball, Topological lattice-ordered groups ............... 1

Stephen Berman, On the low-dimensional cohomology of some

infinite-dimensional simple Lie algebras .................. 27

R. P. Boas and Gerald Thomas Cargo, Level sets of derivatives ......... 37

James K. Deveney and John Nelson Mordeson, Splitting and modularly

perfect fields......................................

Robert Hugh Gilman and Ronald Mark Solomon, Finite groups with small

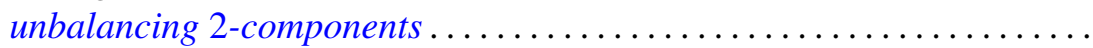

George Grätzer, Andras Hajnal and David C. Kelly, Chain conditions in free products of lattices with infinitary operations..................

Benjamin Rigler Halpern, Periodic points on tori ..................

Dean G. Hoffman and David Anthony Klarner, Sets of integers closed under

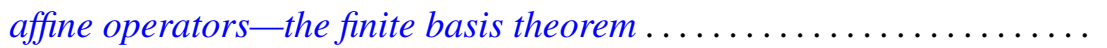

Rudolf-Eberhard Hoffmann, On the sobrification remainder ${ }^{s} X-X \ldots \ldots$

Gerald William Johnson and David Lee Skoug, Scale-invariant

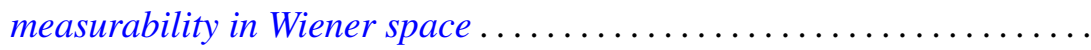

Michael Keisler, Integral representation for elements of the dual of

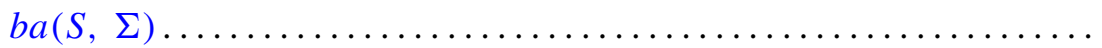

Wayne C. Bell and Michael Keisler, A characterization of the representable Lebesgue decomposition projections ................

Wadi Mahfoud, Comparison theorems for delay differential equations ...

R. Daniel Mauldin, The set of continuous nowhere differentiable functions .

Robert Wilmer Miller and Mark Lawrence Teply, The descending chain condition relative to a torsion theory...

Yoshiomi Nakagami and Colin Eric Sutherland, Takesaki's duality for regular extensions of von Neumann algebras ........ .

William Otis Nowell, Tubular neighborhoods of Hilbert cube manifolds ...

Mohan S. Putcha, Generalization of Lentin's theory of principal solutions of word equations in free semigroups to free product of copies of positive reals under addition

Amitai Regev, A primeness property for central polynomials . ...

Saburou Saitoh, The Rudin kernels on an arbitrary domain. . .

Heinrich Steinlein, Some abstract generalizations of the

Ljusternik-Schnirelmann-Borsuk covering theorem . . . 